Article

\title{
Functional Differential Equations Involving the $\psi$-Caputo Fractional Derivative
}

\author{
Ricardo Almeida
}

Center for Research and Development in Mathematics and Applications (CIDMA), Department of Mathematics, University of Aveiro, 3810-193 Aveiro, Portugal; ricardo.almeida@ua.pt; Tel.: +351-234-370-200

Received: 3 June 2020; Accepted: 22 June 2020; Published: 23 June 2020

check for updates

\begin{abstract}
This paper is devoted to the study of existence and uniqueness of solutions for fractional functional differential equations, whose derivative operator depends on an arbitrary function. The introduction of such function allows generalization of some known results, and others can be also obtained.
\end{abstract}

Keywords: fractional calculus; fractional functional equations

\section{Introduction}

Functional differential equations may be more realistic to model real phenomena, since they take into consideration delays in the problem formulation [1-4]. Thus, they are a natural generalization of the concept of differential equations with delayed arguments, where the change rate of the system depends not only on the current state but is also part of its history. Presently, they are a powerful tool to study a variety of subjects in different fields like Biology, Physics, Engineering, etc. [5-7].

Another possible extension of the previous theory occurs within the framework of fractional calculus. By replacing ordinary derivatives with a fractional derivative, we extend that theory and, in fact, we believe that is a more efficient way to deal with such problems. As is well known, fractional derivatives are non-local operators and, thus, they contain the memory of the past in itself.

In this paper, we study the following fractional functional differential equation (FFDE)

$$
{ }^{C} D_{a+}^{\alpha, \psi} x(t)=f\left(t, x_{t}\right), \quad t \in[a, b]
$$

subject to

$$
x(t)=\phi(t), \quad t \in[a-\varepsilon, a],
$$

where ${ }^{C} D_{a+}^{\alpha, \psi}$ represents the $\psi$-Caputo fractional derivative. By choosing this type of derivative, we present some results valid for a wide class of FFDEs. We find in the literature a vast collection of works dealing with these issues for different fractional derivatives. For example, for the Riemann-Liouville [8-12], the Caputo [13-16], or the Hadamard [17,18] fractional derivatives.

The paper is organized in the following manner. In Section 2, we present some important definitions for our work, and some needed results as well. Then, in Section 3, we study FFDEs and we deal with the question of existence and uniqueness of solution for a FFDE.

\section{Preliminaries}

In this section, we introduce some notations, definitions, and preliminary facts that will be needed in this work. We consider a general form of fractional derivative that involves a function $\psi$. With this new function, we can obtain some well-known definitions of fractional derivatives as particular cases. The fractional order of the derivative is denoted by $\alpha \in \mathbb{R}^{+}$and the kernel by $\psi \in C^{n}([a, b], \mathbb{R})$. 
We assume the $\psi^{\prime}(t)>0$, for all $t \in[a, b]$. Given a function $x:[a, b] \rightarrow \mathbb{R}$, the $\psi$-Caputo fractional integral of $x$, of order $\alpha$, is given by

$$
I_{a+}^{\alpha, \psi} x(t):=\frac{1}{\Gamma(\alpha)} \int_{a}^{t} \psi^{\prime}(\tau)(\psi(t)-\psi(\tau))^{\alpha-1} x(\tau) d \tau .
$$

With respect to differentiation, two kind of operators can be defined: the $\psi$-Riemann-Liouville fractional derivative,

$$
D_{a+}^{\alpha, \psi} x(t):=\left(\frac{1}{\psi^{\prime}(t)} \frac{d}{d t}\right)^{n} I_{a+}^{n-\alpha, \psi} x(t), \quad n=[\alpha]+1,
$$

and the $\psi$-Caputo fractional derivative,

$$
{ }^{C} D_{a+}^{\alpha, \psi} x(t):=D_{a+}^{\alpha, \psi}\left[x(t)-\sum_{k=0}^{n-1} \frac{x_{\psi}^{[k]}(a)}{k !}(\psi(t)-\psi(a))^{k}\right] .
$$

For the last one, we assume that $x \in C^{n-1}([a, b], \mathbb{R}), n$ is given by $n=[\alpha]+1$ if $\alpha \notin \mathbb{N}$, and $n=\alpha$ when $\alpha \in \mathbb{N}$, and $x_{\psi}^{[k]}$ represents

$$
x_{\psi}^{[k]}(t):=\left(\frac{1}{\psi^{\prime}(t)} \frac{d}{d t}\right)^{k} x(t) .
$$

We can easily see that for suitable choices of $\psi$, we obtain some well-known fractional operators, like the Riemann-Liouville, the Caputo, the Hadamard, or the Erdélyi-Kober fractional derivatives [19-24].

When $x \in C^{n}([a, b], \mathbb{R})$, the $\psi$-Caputo fractional derivative can be written as (see [19])

$$
{ }^{C} D_{a+}^{\alpha, \psi} x(t)=I_{a+}^{n-\alpha, \psi} x_{\psi}^{[n]}(t) .
$$

We remark that in such a case, when $\alpha=m \in \mathbb{N}$ is an integer number,

$$
{ }^{C} D_{a+}^{\alpha, \psi} x(t)=x_{\psi}^{[m]}(t),
$$

but when $\alpha \notin \mathbb{N}$,

$$
{ }^{C} D_{a+}^{\alpha, \psi} x(t)=\frac{1}{\Gamma(n-\alpha)} \int_{a}^{t} \psi^{\prime}(\tau)(\psi(t)-\psi(\tau))^{n-\alpha-1} x_{\psi}^{[n]}(\tau) d \tau .
$$

Thus, in opposite to integer order derivatives, fractional derivatives are non-local operators and, for this reason, they keep the memory of the processes. A relation between the fractional integral and the fractional derivative is stated next.

Theorem 1. [25] Let $x:[a, b] \rightarrow \mathbb{R}$ be a continuous function. Then,

$$
{ }^{C} D_{a+}^{\alpha, \psi} I_{a+}^{\alpha, \psi} x(t)=x(t) .
$$

Moreover, if $x \in C^{n-1}([a, b], \mathbb{R})$, then

$$
I_{a+}^{\alpha, \psi C} D_{a+}^{\alpha, \psi} x(t)=x(t)-\sum_{k=0}^{n-1} \frac{x_{\psi}^{[k]}(a)}{k !}(\psi(t)-\psi(a))^{k} .
$$




\section{Fractional Functional Differential Equations}

In this section, we consider a general type of FFDEs, involving the arbitrary function $\psi$. Consider the following FFDE

$$
{ }^{C} D_{a+}^{\alpha, \psi} x(t)=f\left(t, x_{t}\right), \quad t \in[a, b],
$$

subject to

$$
x(t)=\phi(t), \quad t \in[a-\varepsilon, a],
$$

where

1. $\alpha \in(0,1)$ and $\varepsilon>0$ are two fixed real numbers,

2. $f:[a, b] \times C([-\varepsilon, 0], \mathbb{R}) \rightarrow \mathbb{R}$ and $\phi:[a-\varepsilon, a] \rightarrow \mathbb{R}$ are two continuous functions,

3. $x \in C([a-\varepsilon, b], \mathbb{R})$ such that ${ }^{C} D_{a+}^{\alpha, \psi} x$ exists and is continuous on $[a, b]$,

4. $\psi \in C^{1}([a-\varepsilon, b], \mathbb{R})$ such that $\psi^{\prime}(t)>0$, for all $t \in[a-\varepsilon, b]$,

5. $x_{t}:[-\varepsilon, 0] \rightarrow \mathbb{R}$ is the translation $x_{t}(\theta):=x(t+\theta)$.

In these sets, we consider the norms

$$
\|x\|_{[a, b]}=\sup _{t \in[a, b]}|x(t)| \quad \text { and } \quad\left\|x_{t}\right\|_{[-\varepsilon, 0]}=\sup _{\theta \in[-\varepsilon, 0]}\left|x_{t}(\theta)\right| .
$$

We can rewrite our problem in the form of an integral equation.

Theorem 2. A function $x \in C([a-\varepsilon, b], \mathbb{R})$ is a solution to problem (3) and (4) if and only if $x$ satisfies the following equation

$$
x(t)= \begin{cases}\phi(t), & t \in[a-\varepsilon, a] \\ I_{a+}^{\alpha, \psi} f\left(t, x_{t}\right)+\phi(a), & t \in[a, b] .\end{cases}
$$

Proof. First, suppose that $x$ is a solution to (3) and (4). Applying $I_{a+}^{\alpha, \psi}$ to both sides of (3), and taking into consideration Equation (2), we obtain (5). For the converse, given $t \in[a, b]$, applying ${ }^{C} D_{a+}^{\alpha, \psi}$ to (5) and using Equation (1), we obtain (3).

We recall two fundamental results of the fixed-point theory:

Theorem 3. (Banach fixed point theorem) Let $(X, d)$ be a complete metric space. If $F: X \rightarrow X$ is a contraction, then $F$ admits a unique fixed point.

Theorem 4. (Leray-Schauder alternative) Let $E$ be a Banach space, $C$ a closed convex subset of $E$, and $U \subseteq C$ an open subset with $0 \in U$. If $F: \bar{U} \rightarrow C$ is a continuous function and if $F(\bar{U})$ is contained in a compact set, then either

1. F has a fixed point in $\bar{U}$, or

2. there exists $u \in \partial U$ and $\lambda \in(0,1)$ with $u=\lambda F(u)$.

Theorem 5. Suppose that there exists a positive number L such that

$$
|f(t, x)-f(t, y)| \leq L\|x-y\|_{[-\varepsilon, 0]}, \quad \forall t \in[a, b] \forall x, y \in C([-\varepsilon, 0], \mathbb{R})
$$

with

$$
L \frac{(\psi(b)-\psi(a))^{\alpha}}{\Gamma(\alpha+1)}<1 .
$$

Then, there exists a unique solution to problem (3) and (4). 
Proof. Define the set

$$
U:=\left\{x \in C([a-\varepsilon, b], \mathbb{R}):{ }^{C} D_{a+}^{\alpha, \psi} x \text { is continuous in }[a, b]\right\},
$$

and $F: U \rightarrow U$ the operator

$$
F[x](t):= \begin{cases}\phi(t), & \text { if } t \in[a-\varepsilon, a] \\ I_{a+}^{\alpha, \psi} f\left(t, x_{t}\right)+\phi(a), & \text { if } t \in[a, b] .\end{cases}
$$

Let us see that $F$ is well defined. Given $x \in U$, the map $t \mapsto F[x](t)$ is clearly continuous, for all $t \in[a-\varepsilon, b]$. Also, for all $t \in[a, b],{ }^{C} D_{a+}^{\alpha, \psi} F[x](t)=f\left(t, x_{t}\right)$ exists and is continuous. We prove now that $F$ is a contraction map. Let $x, y \in U$ and $t \in[a-\varepsilon, a]$. Then, $|F[x](t)-F[y](t)|=0$. On the other hand, for $t \in[a, b]$, using (6), we have that

$$
\begin{aligned}
|F[x](t)-F[y](t)| & \leq \frac{1}{\Gamma(\alpha)} \int_{a}^{t} \psi^{\prime}(\tau)(\psi(t)-\psi(\tau))^{\alpha-1}\left|f\left(\tau, x_{\tau}\right)-f\left(\tau, y_{\tau}\right)\right| d \tau \\
& \leq \frac{L}{\Gamma(\alpha)} \int_{a}^{t} \psi^{\prime}(\tau)(\psi(t)-\psi(\tau))^{\alpha-1}\left\|x_{\tau}-y_{\tau}\right\|_{[-\varepsilon, 0]} d \tau \\
& \leq L \frac{(\psi(t)-\psi(a))^{\alpha}}{\Gamma(\alpha+1)}\|x-y\|_{[a-\varepsilon, b]} .
\end{aligned}
$$

Therefore, we proved that

$$
\|F[x]-F[y]\|_{[a-\varepsilon, b]} \leq L \frac{(\psi(b)-\psi(a))^{\alpha}}{\Gamma(\alpha+1)}\|x-y\|_{[a-\varepsilon, b]},
$$

that is, $F$ is a contraction map. By the Banach fixed point theorem, $F$ admits a unique fixed point.

Theorem 6. Suppose that there exist

1. two continuous functions $p \in C\left([a, b], \mathbb{R}^{+}\right)$and $q \in C\left(\mathbb{R}_{0}^{+}, \mathbb{R}_{0}^{+}\right)$such that $q$ is nondecreasing and the following condition holds:

$$
|f(t, x)| \leq p(t) q\left(\|x\|_{[-\varepsilon, 0]}\right), \quad \forall t \in[a, b] \forall x \in C([-\varepsilon, 0], \mathbb{R}),
$$

2. a positive constant $M \in \mathbb{R}$ such that

$$
\frac{\|p\|_{[a, b]} q(M)(\psi(b)-\psi(a))^{\alpha}+|\psi(a)| \Gamma(\alpha+1)}{M \Gamma(\alpha+1)}<1 \text { and } M>\sup _{t \in[a-\varepsilon, a]}|\phi(t)| .
$$

Then problem (3) and (4) possesses at least one solution in $[a-\varepsilon, b]$.

Proof. Let $F$ be the function defined in (8). The proof will be divided in several steps.

1. Function $F$ is continuous. 
Let $\left(x_{n}\right)$ be a sequence in $C([a-\varepsilon, b], \mathbb{R})$ whose limit is $x \in C([a-\varepsilon, b], \mathbb{R})$. Then, for all $t \in[a, b]$, we have the following:

$$
\begin{aligned}
\left|F\left[x_{n}\right](t)-F[x](t)\right| & \leq \frac{1}{\Gamma(\alpha)} \int_{a}^{t} \psi^{\prime}(\tau)(\psi(t)-\psi(\tau))^{\alpha-1}\left|f\left(\tau, x_{n \tau}\right)-f\left(\tau, x_{\tau}\right)\right| d \tau \\
& \leq \frac{1}{\Gamma(\alpha)} \int_{a}^{t} \psi^{\prime}(\tau)(\psi(t)-\psi(\tau))^{\alpha-1} \sup _{t \in[a, b]}\left|f\left(t, x_{n t}\right)-f\left(t, x_{t}\right)\right| d \tau \\
& \leq \sup _{t \in[a, b]}\left|f\left(t, x_{n t}\right)-f\left(t, x_{t}\right)\right| \frac{(\psi(b)-\psi(a))^{\alpha}}{\Gamma(\alpha+1)},
\end{aligned}
$$

and the last term converges to zero as $n$ goes to infinity, since $f$ is a continuous function.

2. Function $F$ is uniformly bounded.

Let

$$
\bar{B}_{R}:=\left\{x \in U:\|x\|_{[a-\varepsilon, b]} \leq R\right\},
$$

where $R>0$ is a real number. Given a function $x \in \bar{B}_{R}$ and $t \in[a, b]$ (the case when $t \in[a-\varepsilon, a]$ is clear):

$$
\begin{aligned}
|F[x](t)| & \leq \frac{1}{\Gamma(\alpha)} \int_{a}^{t} \psi^{\prime}(\tau)(\psi(t)-\psi(\tau))^{\alpha-1}\left|f\left(\tau, x_{\tau}\right)\right| d \tau \\
& \leq \frac{1}{\Gamma(\alpha)}\|p\|_{[a, b]} q\left(\|x\|_{[a-\varepsilon, b]}\right) \int_{a}^{t} \psi^{\prime}(\tau)(\psi(t)-\psi(\tau))^{\alpha-1} d \tau \\
& \leq \frac{(\psi(b)-\psi(a))^{\alpha}}{\Gamma(\alpha+1)}\|p\|_{[a, b]} q\left(\|x\|_{[a-\varepsilon, b]}\right) \\
& \leq \frac{(\psi(b)-\psi(a))^{\alpha}}{\Gamma(\alpha+1)}\|p\|_{[a, b]} q(R),
\end{aligned}
$$

which does not depend on $x$. Thus, $F$ maps bounded sets into bounded sets of $C([a-\varepsilon, b], \mathbb{R})$.

3. Function $F$ maps bounded sets into equicontinuous sets.

Let us prove that $F\left(\bar{B}_{R}\right)$ is equicontinuous. For that purpose, consider $t_{1}, t_{2} \in[a, b]$, with $t_{1}>t_{2}$, and $x \in \bar{B}_{R}$. Then,

$$
\begin{aligned}
\left|F[x]\left(t_{1}\right)-F[x]\left(t_{2}\right)\right|= & \mid \frac{1}{\Gamma(\alpha)} \int_{a}^{t_{1}} \psi^{\prime}(\tau)\left(\psi\left(t_{1}\right)-\psi(\tau)\right)^{\alpha-1} f\left(\tau, x_{\tau}\right) d \tau \\
& -\frac{1}{\Gamma(\alpha)} \int_{a}^{t_{2}} \psi^{\prime}(\tau)\left(\psi\left(t_{2}\right)-\psi(\tau)\right)^{\alpha-1} f\left(\tau, x_{\tau}\right) d \tau \mid \\
\leq & \frac{1}{\Gamma(\alpha)}\left|\int_{a}^{t_{2}} \psi^{\prime}(\tau)\left[\left(\psi\left(t_{1}\right)-\psi(\tau)\right)^{\alpha-1}-\left(\psi\left(t_{2}\right)-\psi(\tau)\right)^{\alpha-1}\right] f\left(\tau, x_{\tau}\right) d \tau\right| \\
& +\frac{1}{\Gamma(\alpha)}\left|\int_{t_{2}}^{t_{1}} \psi^{\prime}(\tau)\left(\psi\left(t_{1}\right)-\psi(\tau)\right)^{\alpha-1} f\left(\tau, x_{\tau}\right) d \tau\right| \\
\leq & \frac{\|p\|_{[a, b]} q(R)}{\Gamma(\alpha+1)}\left[\left|\left(\mid \psi\left(t_{1}\right)-\psi(a)\right)^{\alpha}-\left(\psi\left(t_{2}\right)-\psi(a)\right)^{\alpha}-\left(\psi\left(t_{1}\right)-\psi\left(t_{2}\right)\right)^{\alpha}\right|\right. \\
& \left.+\left(\psi\left(t_{1}\right)-\psi\left(t_{2}\right)\right)^{\alpha}\right]
\end{aligned}
$$

which converges to zero as $t_{2} \rightarrow t_{1}$. Let us observe that for $t_{1}, t_{2} \in[a-\varepsilon, a]$,

$$
\left|F[x]\left(t_{1}\right)-F[x]\left(t_{2}\right)\right|=\left|\phi\left(t_{1}\right)-\phi\left(t_{2}\right)\right| \rightarrow 0, \quad \text { when } t_{2} \rightarrow t_{1},
$$


and for $t_{1} \in[a, b]$ and $t_{2} \in[a-\varepsilon, a]$,

$$
\left|F[x]\left(t_{1}\right)-F[x]\left(t_{2}\right)\right|=\left|\frac{1}{\Gamma(\alpha)} \int_{a}^{t_{1}} \psi^{\prime}(\tau)\left(\psi\left(t_{1}\right)-\psi(\tau)\right)^{\alpha-1} f\left(\tau, x_{\tau}\right) d \tau+\phi(a)-\phi\left(t_{2}\right)\right|
$$

also converges to zero as $t_{2} \rightarrow t_{1}(\rightarrow a)$. By the Ascoli-Arzela Theorem, $F\left(\bar{B}_{R}\right)$ is contained in a compact set.

4. Let

$$
B_{M}:=\left\{x \in U:\|x\|_{[a-\varepsilon, b]}<M\right\} .
$$

Let us see that the function $F: \overline{B_{M}} \rightarrow \overline{B_{M}}$ is well defined. Let $x \in \overline{B_{M}}$. For $t \in[a-\varepsilon, a]$,

$$
|F[x](t)|=|\phi(t)|<M,
$$

and for $t \in[a, b]$,

$$
|F[x](t)| \leq \frac{\|p\|_{[a, b]} q(M)}{\Gamma(\alpha+1)}(\psi(b)-\psi(a))^{\alpha}+|\phi(a)|<M
$$

under assumption (9).

To end the proof, we will see that condition 2 in Theorem 4 cannot be satisfied. Given $x \in B_{M}$ and $t \in[a-\varepsilon, b]$, we have that $|F[x](t)|<M$. If there were a function $x \in \partial B_{M}$ and a real number $\lambda \in(0,1)$ such that $x=\lambda F[x]$, then we would have

$$
M=\|x\|_{[a-\varepsilon, b]}=\lambda\|F[x]\|_{[a-\varepsilon, b]}<M
$$

obtaining, therefore, a contradiction.

\section{Example}

Consider the following FFDE

$$
{ }^{C} D_{a+}^{\alpha, \psi} x(t)=\frac{2}{\Gamma(3-\alpha)} \sqrt{\left\|x_{t}\right\|_{[-\varepsilon, 0]}}(\psi(t)-\psi(a))^{1-\alpha}, \quad t \in[a, b],
$$

subject to

$$
x(t)=0, \quad t \in[a-\varepsilon, a] .
$$

The solution to (10) and (11) is the function

$$
\bar{x}(t)= \begin{cases}(\psi(t)-\psi(a))^{2}, & t \in[a, b] \\ 0, & t \in[a-\varepsilon, a] .\end{cases}
$$

\section{Conclusions}

In this paper, we present some results on existence and uniqueness of solutions for fractional functional differential equations with dependence on a general form of derivative that depends on an arbitrary kernel. For suitable choices of $\psi$, some well-known results for fractional operators, like the Riemann-Liouville, the Caputo, the Hadamard, or the Erdélyi-Kober fractional derivatives, are obtained. In addition, a correct kernel choice is essential when modeling real phenomena, to fit theoretical models to real data, as was shown in [26]. 
For future work, applications and fundamental results of FFDE (3) and (4) should be studied. Also, it would be interesting to develop numerical methods for this type of problem, similar to the one presented in [27] for fractional differential equations.

Funding: Work supported by Portuguese funds through the CIDMA-Center for Research and Development in Mathematics and Applications, and the Portuguese Foundation for Science and Technology (FCT-Fundação para a Ciência e a Tecnologia), within project UIDB/04106/2020.

Conflicts of Interest: The author declares no conflict of interest.

\section{References}

1. Dong, Q.; Fan, Z.; Li, G. Existence of solutions to nonlocal neutral functional differential and integrodifferential equations. Int. J. Nonlinear Sci. 2008, 5, 140-151.

2. Hernández, E.; Henríquez, H.R. Existence results for partial neutral functional differential equations with unbounded delay. J. Math. Anal. Appl. 1998, 221, 452-475. [CrossRef]

3. Hernández, E.; Henríquez, H.R. Existence of periodic solutions of partial neutral functional differential equations with unbounded delay. J. Math. Anal. Appl. 1998, 221, 499-522. [CrossRef]

4. Ye, R.; Dong, Q.; Li, G. Existence of solutions for double perturbed neutral functional evolution equation. Int. J. Nonlinear Sci. 2009, 8, 360-367.

5. Hale, J.; Lunel, S.M.V. Introduction to Functional Differential Equations. In Applied Mathematical Sciences; Springer: New York, NY, USA, 1993; Volume 99.

6. Kolmanovskii, V.; Myshkis, A. Introduction to the Theory and Applications of Functional Differential Equations. In Mathematics and Its Applications; Kluwer Academic Publishers: Dordrecht, The Netherland, 1999; Volume 463.

7. Kolmanovskii, V.B.; Nosov, V.R. Stability of Functional Differential Equations; Academic Press: New York, NY, USA, 1986.

8. Belarbi, A.; Benchohra, M.; Ouahab, A. Uniqueness results for fractional functional differential equations with infinite delay in Fréchet spaces. Appl. Anal. 2006, 85, 1459-1470. [CrossRef]

9. Benchohra, M.; Henderson, J.; Ntouyas, S.K.; Ouahab, A. Existence results for fractional order functional differential equations with infinite delay. J. Math. Anal. Appl. 2008, 338, 1340-1350. [CrossRef]

10. Darwish, M.A.; Ntouyas, S.K. Existence results for a fractional functional differential equation of mixed type. Commun. Appl. Nonlinear Anal. 2008, 15, 47-55.

11. Henderson, J.; Ouahab, A. Fractional functional differential inclusions with finite delay. Nonlinear Anal. 2009, 70, 2091-2105. [CrossRef]

12. Lakshmikantham, V. Theory of fractional functional differential equations. Nonlinear Anal. 2008, 69, 3337-3343. [CrossRef]

13. Chen, F.; Chen, A.; Wang, X. On the solutions for impulsive fractional functional differential equations. Differ. Equ. Dynam. Syst. 2009, 17, 379-391. [CrossRef]

14. Chen, F.; Zhou, Y. Attractivity of fractional functional differential equations. Comput. Math. Appl. 2011, 62, 1359-1369. [CrossRef]

15. El-Sayed, A.M.A. Nonlinear functional differential equations of arbitrary orders. Nonlinear Anal. 1998, 33, $181-186$. [CrossRef]

16. Guo, T.L.; Jiang, W. Impulsive fractional functional differential equations. Comput. Math. Appl. 2012, 64, $3414-3424$. [CrossRef]

17. Ahmad, B.; Ntouyas, S.K. Initial value problems of fractional order Hadamard-type functional differential equations. Electron. J. Differ. Equ. 2015, 77, 1-9.

18. Agarwal, R.P.; Ntouyas, S.K.; Ahmad, B.; Alzahrani, A.K. Hadamard-type fractional functional differential equations and inclusions with retarded and advanced arguments. Adv. Differ. Equ. 2016, 2016, 92. [CrossRef]

19. Almeida, R. A Caputo fractional derivative of a function with respect to another function. Commun. Nonlinear Sci. Numer. Simul. 2017, 44, 460-481. [CrossRef]

20. Gambo, Y.Y.; Jarad, F.; Baleanu, D.; Abdeljawad, T. On Caputo modification of the Hadamard fractional derivatives. Adv. Differ. Equ. 2014, 10. [CrossRef]

21. Jarad, F.; Abdeljawad, T.; Baleanu, D. Caputo-type modification of the Hadamard fractional derivatives. Adv. Differ. Equ. 2012, 142. [CrossRef] 
22. Kilbas, A.A.; Srivastava, H.M.; Trujillo, J.J. Theory and Applications of Fractional Differential Equations. In North-Holland Mathematics Studies; Elsevier Science B.V.: Amsterdam, Switzerland, 2006; Volume 204.

23. Luchko, Y.; Trujillo, J.J. Caputo-type modification of the Erdélyi-Kober fractional derivative. Fract. Calc. Appl. Anal. 2007, 10, 249-267.

24. Samko, S.G.; Kilbas, A.A.; Marichev, O.I. Fractional Integrals and Derivatives, Translated from the 1987 Russian Original; Gordon and Breach: Yverdon, Switzerland, 1993.

25. Almeida, R.; Malinowska, A.B.; Monteiro, M.T.T. Fractional differential equations with a Caputo derivative with respect to a kernel function and their applications. Math. Meth. Appl. Sci. 2018, 41, 336-352. [CrossRef]

26. Almeida, R. What is the best fractional derivative to fit data? Appl. Anal. Discrete Math. 2017, 11, 358-368. [CrossRef]

27. Asanov, A.; Almeida, R.; Malinowska, A.B. Fractional differential equations and Volterra-Stieltjes integral equations of the second kind. Comput. Appl Math. 2019, 38, 160. [CrossRef]

(C) 2020 by the author. Licensee MDPI, Basel, Switzerland. This article is an open access article distributed under the terms and conditions of the Creative Commons Attribution (CC BY) license (http:/ / creativecommons.org/licenses/by/4.0/). 